Review

\title{
Cellular membrane affinity chromatography (CMAC) in drug discovery from complex natural matrices
}

\author{
Cayman Stephen ${ }^{1}$, Abdelfatteh El Omri ${ }^{2}$, Lukasz Ciesla*1 \\ ${ }^{1}$ Department of Biological Sciences, The University of Alabama, Science and Engineering Complex 2329 \\ Tuscaloosa, AL 35487, USA \\ ${ }^{2}$ Genomics and Biotechnology Section and Research Group, Department of Biological Sciences, Faculty of Science, King \\ Abdulaziz University (KAU), Jeddah 21589, Box 80141, Saudi Arabia
}

*Corresponding Author: E-mail: Imciesla@ua.edu; Tel.: +1-205-348-1828;

Received: April 08, 2018; Revised: May 06, 2018; Published: May 21, 2018

\begin{abstract}
Secondary plant metabolites are evolutionary-designed molecules that interact with multiple biological targets in human organisms. Identification of pharmacologically active phytochemicals is usually a time consuming and costly process. Cellular membrane affinity chromatography (CMAC) allows the detection of secondary metabolites present in complex natural matrices, e.g. plant extracts and their interactions with the immobilized fully-functional transmembrane proteins. After the isolation process of the binding compounds, CMAC columns can be used to study the binding process between the potential new ligands and the immobilized transmembrane protein target. The following parameters can be determined using CMAC columns: binding affinity $\left(K_{\mathrm{d}}\right)$, association rate constant $\left(k_{\mathrm{on}}\right)$, dissociation rate constant $\left(k_{\text {off }}\right)$ and the equilibrium constant for complex formation (K). This review summarizes the preparation steps and the use of CMAC columns in the drug discovery process of new potential drug leads present in complex natural matrices.
\end{abstract}

\section{Keywords}

artificial membrane stationary phase; bioassay; chromatography; frontal affinity chromatography; IAM.PC; immobilized protein; phytochemicals; plant extracts

\section{Introduction}

For thousands of years, humankind has relied on remedies obtained from nature, mostly from plants. Secondary plant metabolites can be considered as evolutionary designed drugs, that have evolved as a means of herbal communication with other organisms. Plants use the array of phytochemicals that serve multiple purposes, for example: protection from abiotic stress, like excessive UV radiation (polyphenols, terpenes and terpenoids), attraction of pollinators (volatile terpenes), warding off herbivores (noxious phytochemicals, e.g. alkaloids), protection from bacterial or fungal infections (polyphenols, terpenoids, etc) [1-4]. Herbivores evolved different forms of biochemical changes, that protect them from the deleterious effects of some of the phytochemicals, for example: CYP450 superfamily of enzymes, transporter proteins and stress-response cellular signalling pathways [1-4]. Many of these mechanisms are highly conserved adaptive stress response systems that humans share with simple herbivores. Numerous secondary 
metabolites can be considered as already optimized drug leads targeting specific receptors, enzymes or biochemical processes [5]. The huge potential of plant-derived drugs is exemplified by numerous secondary plant metabolites currently used in clinics, for example, artemisinin, ivermectin, morphine, digitoxin, vincristine, podophyllotoxin, and many others [6].

Unfortunately, plants do not reveal their secrets easily as the identification of pharmacologically active phytochemicals is usually a difficult and time-consuming process [7]. Many pharmaceutical companies have abandoned drug discovery from natural products, considering it as a demanding high resource commitment and often leading to disappointing results (identification of known compounds and poor biological activity). Technical problems also prevent now-commonly used high-throughput screening (HTS) techniques from testing complex natural matrices for pharmacologically active compounds [8]. HTS techniques are designed to test huge libraries of individual compounds. In cases of complex samples, only a simple answer would be delivered if the HTS approach was used: the extract is active or not active. Identification of pharmacologically active metabolites would have to follow the HTS approach.

In the traditional approach to drug discovery from natural samples, the first step usually involves the isolation of individual compounds. This step often leads to obtaining compounds which are easily isolated with reasonable quantities needed to run biological activity assays. The most abundant secondary metabolites are usually isolated and many of them have already well-established and known pharmacological activity profiles [7]. The use of bio-guided fractionation has provided some solutions by narrowing down the isolation process to subfractions that have been identified as having the highest activity. However, bio-guided fractionation still requires repetitive multiple steps to identify the subfractions that are rich in biologically active compounds.

Different attempts have been made to shorten the process of drug lead identification from plant extracts. Many of these attempts focused on developing target-oriented assays enabling identification of compounds with desired activity. Secondary metabolites act by interacting with numerous targets in the human body, for example, cytosolic proteins or transmembrane receptors [5]. These new assays are designed to screen complex matrices and identify compounds that selectively interact with the biological target (enzyme, receptor) [7]. Development of target-oriented assays with immobilized functional enzymes has been more popular than the attempts to design tests aimed at identification of compounds targeting cell membrane proteins. Cytosolic proteins are usually covalently bonded using their $\mathrm{N}$ - or C-terminus groups forming the covalent bond with chemical moieties on the surface of the assay scaffold, e.g. the inside of the column or magnetic beads $[9,10]$. Many natural compounds target cell membrane proteins but the solutions developed for cytosolic proteins cannot be used to immobilize transmembrane proteins [7]. This review focuses on the development of novel bioassays with the immobilized fully functional cell membrane proteins and their use in drug leads discovery from complex natural matrices.

One of the most important issues in developing assays targeting transmembrane receptors is to make sure functional protein is immobilized. Introducing chemical changes in the immobilization process of enzymes may lead to irreversible modifications to protein structure and result in failure to develop functional assay [11]. It is important to keep boundary lipids surrounding the transmembrane protein intact as the subtle interactions between transmembrane domains and these lipids influences how the potential ligands bind to the protein. It must be remembered that in the process of transmembrane protein immobilization, the cell membrane fragments and not the pure proteins are immobilized [7]. Stripping off the boundary lipids would not only lead to changing the receptor binding activity but also would probably result in the immobilization of protein aggregates, that would form during the 
immobilization process [11]. The aggregates would develop because nonpolar amino acid residues, forming the transmembrane domains would bury in the interior of the aggregates not to get exposed to an aqueous environment. The immobilization of cell membrane fragments with functional transmembrane receptors can be achieved in the process of cellular membrane affinity chromatography (CMAC) column preparation [11]. Successful immobilization of transmembrane fragments will result in obtaining an assay that enables the study of ligand-receptor interactions in close to physiological conditions (Figure 1).

\section{Cellular membrane affinity chromatography columns - a short historical perspective}

To the best of our knowledge, Lundahl's group was the first to successfully immobilize transmembrane protein, the glucose transporter, GLUT 1 [12]. This study stimulated Wainer's group to approach the immobilization process of nicotinic acetylcholine receptors (nAChRs) on lipid monolayers [13]. Interestingly $\alpha_{3} \beta_{4}$ nAChRs were also successfully immobilized on the immobilized artificial membrane (IAM) stationary phase, developed by Pidgeon et al. [14] and currently available from Regis Technologies, Figure 2.

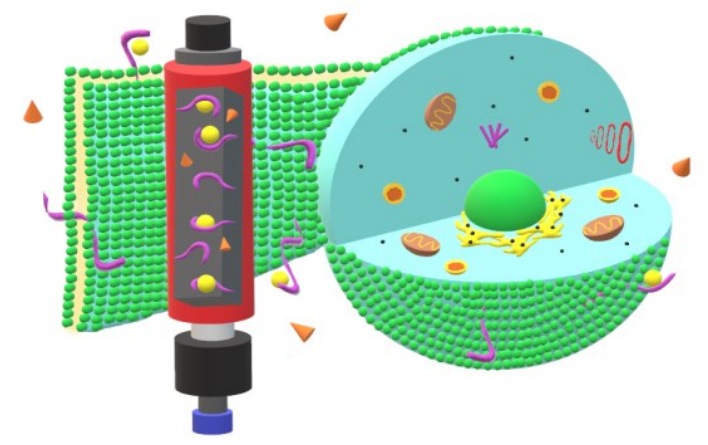

Figure 1. Cellular membrane affinity chromatography column contains cell membrane fragments with fully functional transmembrane protein (TPM) targets and therefore allows for studying the interactions between small molecules and TMP in close to physiological conditions

Studies by the Wainer group showed that CMAC prepared with the use of IAM particles were characterized by better reproducibility compared to liposomal columns [13]. Since the successful immobilization of nAChRs on the IAM stationary phase, multiple transmembrane proteins have been used to prepare CMACs with IAM.PC (immobilized artificial membrane phosphatidylcholine). The examples of transmembrane proteins immobilized on IAM.PC particles include: ligand-gated ion channels $\left(\alpha_{x} \beta_{y} n A C h R x\right.$ $=3,4,7 ; y=2,3,4 ; \mathrm{GABA}_{\mathrm{A}}$, NMDA, combination of $\mathrm{nAChR}$, NMDA) [11, 13, 15-30], G-protein coupled receptors (opioid, $\beta$-adrenergic, $\mathrm{P} 2 \mathrm{Y} 1$, Histamine 1-4, $\mathrm{CB}_{1}, \mathrm{CB}_{2}$ ) [31-35] and drug transporters (ABC: Pgp, MRP1, MRP2, BCRP; SLC: hOCT and hOCT SNPs, hOAT) [36-39]. IAM.PC mimics the cell membrane environment and keeps the experimental conditions close to the physiological state. The surface of the IAM.PC stationary phase still contains residual silanol and amine groups on its surface, which may become charged during the analysis, resulting in changed retention parameters of the studied ligands. A new class of stationary phase has been developed by Regis, IAM.PC.DD2 particles with amine groups endcapped, Figure 2. The endcapping leads to an increase of the stationary phase stability and its lipophilicity. To the best of our knowledge, there are no data available on the possible use of IAM.PC.DD2 particles in the preparation of CMACs. Future experiments should determine whether endcapping the residual amine and silanol groups would result in more reproducible data generated with CMACs. Possible problems with the use of more lipophilic artificial membrane particles are discussed further in this review. 


\section{IAM.PC}

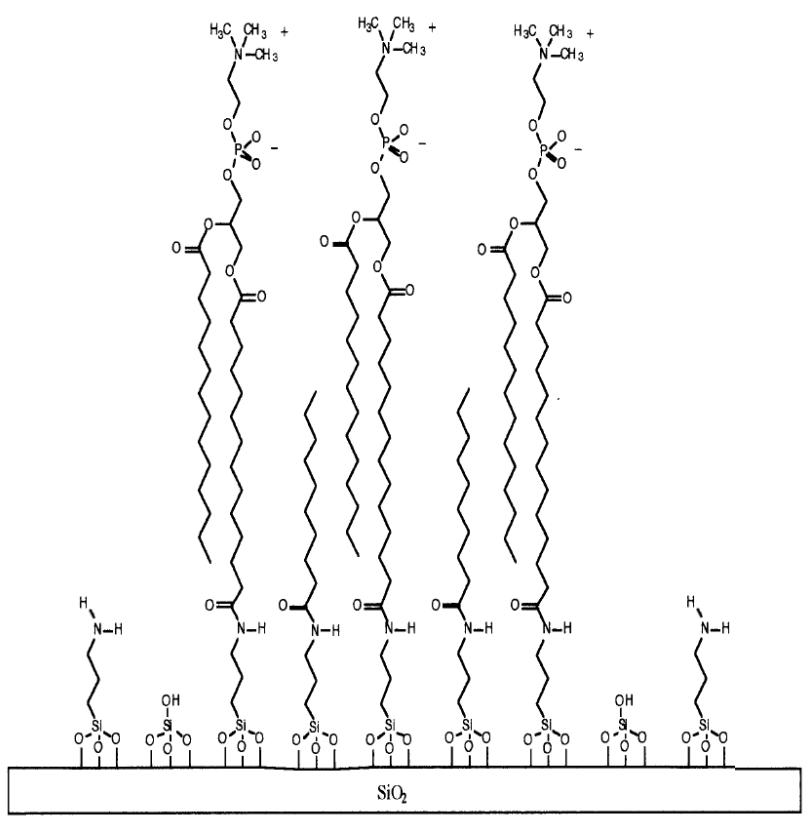

\section{IAM.PC.DD2}

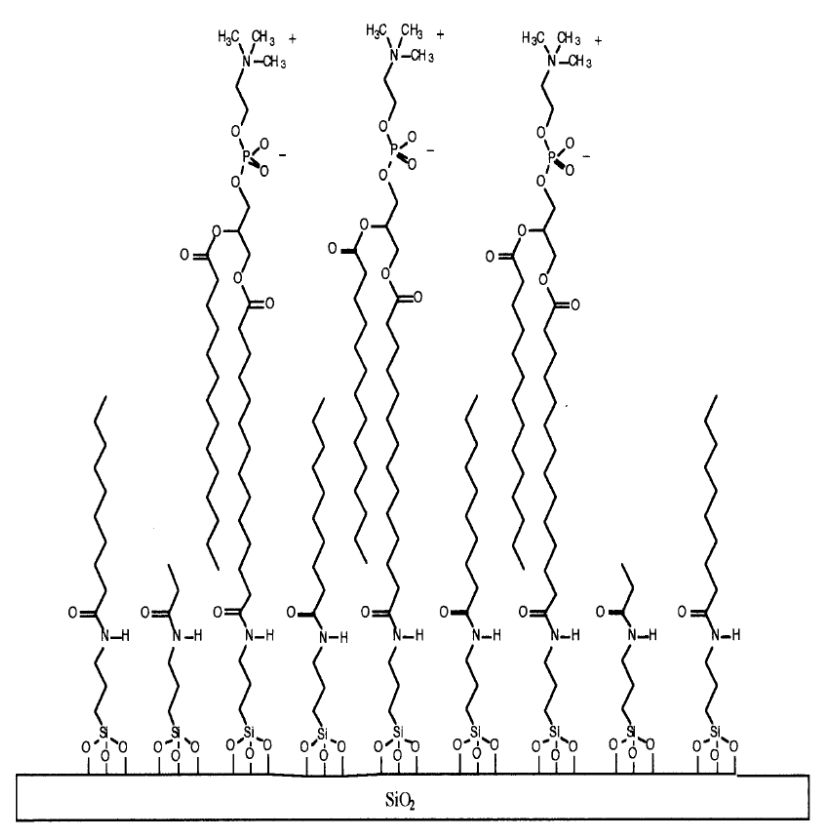

Figure 2. Differences in surface chemistry of IAM.PC and IAM.PC.DD2 particles. With permission from Regis Technologies

Another approach developed to study ligand-protein interactions involves the use of activated silica for the adsorption of cell membrane fragments and is called cell membrane chromatography (CMC) [40]. The use of silica introduces silica groups in the process of studying ligand-protein interactions, which does not closely resemble physiological conditions. However, numerous drug discovery approaches utilizing CMCs have been reported in the literature, for example [40-43]. This review purely focuses on the approach with the artificial membrane as the support for the adsorbing cell membrane fragments and therefore $\mathrm{CMC}$ is not further discussed in this paper.

\section{Preparation steps of cellular membrane affinity chromatography columns}

The process of preparing CMAC columns has been described in detail elsewhere by Moaddel and Wainer [11]. In this review, critical steps of column preparation are described and particular attention is paid to issues that have the most critical impact on the process of developing a functional CMAC. The process of CMAC development can be divided into 5 consecutive steps: (1) transmembrane protein source cell/tissue homogenization, (2) solubilization, (3) dialysis and immobilization, (4) packing and (5) column characterization (Figure 3).

\section{Homogenization}

The first step in preparing a CMAC column is the choice of transmembrane protein source (cell line or tissue). If CMAC is to be used in the drug discovery screening process of complex natural matrices then the use of transfected cell line overexpressing the transmembrane protein of choice is recommended. A CMAC column prepared with a transfected cell line will result in a higher number of active sites increasing the capacity and selectivity of the new column [11]. However, if the experiments using CMAC focus on the characterization of transmembrane protein and its interaction with particular ligands, the use of a native cell line is advised. The use of native cell line guarantees that the studied protein is surrounded by the boundary lipids that assure its full natural functionality. In native cell lines, transmembrane proteins also 
may undergo some specific post-translational modifications that may not take place in the case of a transfected cell line.

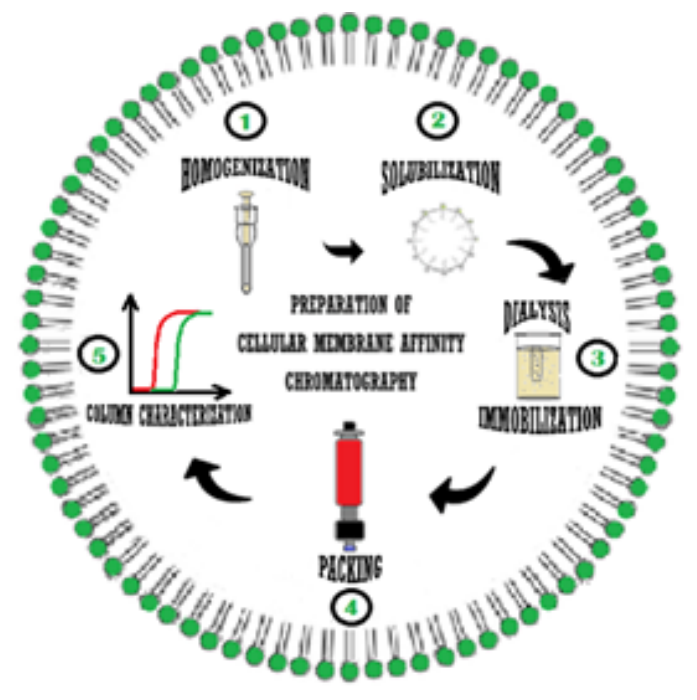

Figure 3. Preparation steps of cell membrane affinity chromatography columns (CMAC)

The homogenization process is performed in a buffered environment (usually Tris $-\mathrm{HCl}$ buffer, $50 \mathrm{mM}$, $\mathrm{pH}$ 7.4) with the addition of salts, to keep it close to physiological conditions and protect the transmembrane protein from degradation. The homogenization procedures are performed on ice and all centrifugation steps are executed at $4^{\circ} \mathrm{C}$. Glass-dounce homogenizers, polytron homogenizers or sonicators are commonly used in the process of cell homogenization [11]. The use of protease and peptidase inhibitors is required to immobilize functional transmembrane protein. The choice of proper inhibitors depends on the type of cell line used in the experiments. The number of cells used in the experiment depends on the level of protein expression and may vary from 1 million to 50 million cells per pellet. After rupturing the cell structure, the resulting homogenate is centrifuged at low speed to remove nuclei and the pellet is discarded. The remaining supernatant is subsequently centrifuged at high speed and the pellet containing cell membranes is collected. In some cases, the use of additional reagents is required for immobilization of functional protein. For example, the addition of glycerol enhances the stability of the protein in aqueous solutions and was found to be useful in some CMAC preparation protocols [11]. As previously mentioned the interactions of protein transmembrane domains with boundary lipids influences the activity of the protein. The addition of some phospholipids in the buffer solutions may be required in some instances, as previously reported [11]. For more experimental details please refer to the manuscript by Moaddel and Wainer [11].

\section{Solubilization}

The protein isolation is completed using the solubilization step. As in the previous homogenization stage, all the experiments are performed at low temperature $\left(4^{\circ} \mathrm{C}\right)$ in a buffered environment. Compared to the homogenization buffer, used in step 1 , solubilization buffer contains detergent, that solubilizes transmembrane proteins by forming micelles around them. Several parameters need to be considered when choosing the detergent. Again, it is of crucial importance to remember that the goal is to immobilize cell membrane fragments with transmembrane proteins and not the purified proteins. The use of strong detergents, like Tween or Triton X-100, should be avoided as it may lead to stripping off the boundary lipids resulting in the immobilization of nonactive proteins or protein aggregates. These detergents would also cause problems in the next step, that requires elimination of the detergent, as they are difficult to 
remove using dialysis. Previous studies have shown the use of the following detergents results in the immobilization of functional transmembrane proteins: sodium cholate (optimal for immobilization of ligand-gated ion channels), CHAPS (works well with GPCRs), and $\beta$-octyl glucopyranoside [11]. The pellet collected after the completion of the homogenization step is mixed with the solubilization buffer and left overnight on the orbital shaker at a temperature of $4^{\circ} \mathrm{C}$.

\section{Dialysis and immobilization}

After the completion of the overnight solubilization step, the mixture is centrifuged at high speed and the pellet discarded. At this point, the cell membrane fragments are in the supernatant "trapped" in the micelles formed by the detergent. The resulting supernatant is put in contact with IAM.PC particles and left on the orbital shaker for 1 hour. To complete the process of immobilization of the cell membrane fragments on the IAM particles, the mixture containing IAM.PC stationary phase and micelles are placed in dialysis tubing and dialysed for 48 hours, to get rid of the detergent. Disruption of micelles results in freeing the cell membrane fragments and the phospholipids forming cellular bilayer adsorb on IAM particles to protect lipophilic carbon tails from their exposure to the aqueous environment.

\section{Packing}

After completing the dialysis step the content of the dialysis tubing is centrifuged at low speed and the supernatant is discarded. The remaining pellet is washed three times with ammonium acetate buffer (10 $\mathrm{mM}, \mathrm{pH}$ 7.4). Ammonium acetate buffer is used also as running buffer, as it is compatible with mass spectrometers, commonly used as detectors in subsequent experiments involving the use of CMAC. The use of Tris- $\mathrm{HCl}$ buffer would result in an additional Tris peak ( $\mathrm{m} / \mathrm{z} 122.1$, positive ionization mode) in the chromatograms. Following the three washes, the pellet is suspended in $1 \mathrm{~mL}$ of ammonium acetate buffer and packed in a glass column to produce a transmembrane protein IAM.PC CMAC column TMP-CMAC (Figure 4). The packing procedure is a low-pressure packing and therefore does not require any sophisticated equipment. The packing process resembles the slurry pack procedure used in classical column preparation for the isolation of secondary metabolites, but on a smaller scale. After packing the column should be washed with running buffer (ammonium acetate, $10 \mathrm{mM}, \mathrm{pH} 7.4$ ) at the flow rate of approximately $0.2 \mathrm{ml} / \mathrm{min}$.

\section{Column characterization and usage}

After completing all the preparation steps, the moment of truth comes: testing the column to check if functional receptors are immobilized on the surface of the IAM particles. This can be achieved through several experimental ways. Firstly, the immobilization of transmembrane proteins onto IAM particles can be confirmed using confocal microscopy [44]. IAM with immobilized cell membrane fragments is incubated with a ligand known to bind to the target protein. The ligand used in the study should contain fluorochrome for the identification with the confocal microscope. IAM particles should also be coincubated with a series of concentrations of another ligand, binding to the same site of the investigated receptor, to confirm the competitive displacement of the ligand containing fluorochrome.

In another set of experiments confirming the immobilization of functional receptors onto IAM particles an artificial mixture of known binders and non-binders is injected onto the column. Known binders should be retained on the column with the immobilized receptors significantly longer compared to a negative control column CMAC (-). The concept of negative control columns is fully explained in the next section of this article discussing the use of CMAC columns in drug discovery from natural samples. 


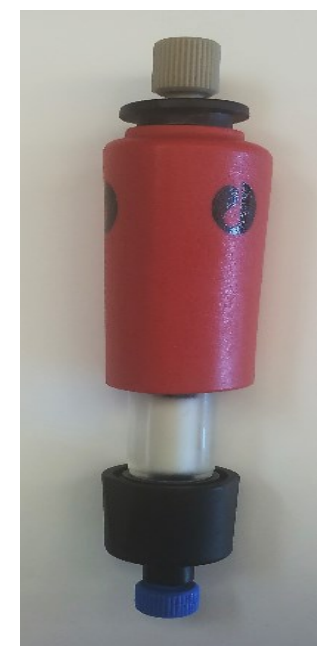

Figure 4. Cell membrane affinity chromatography column after packing IAM particles with the immobilized cell membrane fragments into a glass column

Finally, a series of concentrations of a known high-affinity ligand should be injected in consecutive runs onto the CMAC column to determine the compound's binding affinity $\left(K_{D}\right)[11]$. The binding affinity value is obtained using frontal affinity chromatography (FAC) and a detector sensitive enough to detect the marker ligand at the used concentration. Mass spectrometers are most commonly used in this approach, but any other detector suitable for the detection of the marker ligand may be applied. The concept of frontal affinity chromatography was thoroughly reviewed in the paper by $\mathrm{Ng}$ et al. [45] and will only be shortly discussed in this review. Frontal affinity chromatography is a biophysical method to study the interactions between a ligand present in the mobile phase and the immobilized target molecule, e.g. a receptor [45]. A known marker ligand (for example epibatidine for nAChRs) at constant concentration is run through the column with the immobilized transmembrane protein, producing a characteristic sigmoidal breakthrough curve (Figure 5). At the beginning of the analysis, the number of available binding sites (immobilized transmembrane protein) is higher than the number of the titrating molecules of the marker ligand (the flat bottom portion of the curve). After all the binding sites are saturated with the ligand, the concentration of the ligand leaving the column gradually increases producing the breakthrough. At the end of the analysis, the concentration of the ligand entering and eluting from the column is identical as illustrated by the plateau portion of the curve. The inflexion point depends on the ligand concentration, the number of available binding sites and the compound's binding affinity [45]. Typical chromatograms using FAC obtained for orthosteric (a) and allosteric (b) nAChR binding sites are presented in Figure 5 [30].

Using frontal affinity chromatography with CMAC columns was proved not only to be useful in characterization and studies involving the orthosteric but also with the allosteric binding sites of the immobilized transmembrane protein [30]. Frontal displacement chromatography can be applied to determine the binding affinity of unknown ligand binding to the same site as the marker ligand. It is done by injecting constant concentration of the marker ligand with varying concentrations of the studied displacer and using the following equation [11]:

$$
[D i s]\left(V-V_{\text {min }}\right)=P[D i s]\left(K_{d D i s}+[D i s]\right)^{-1}
$$

where: [Dis] - concentration of the displacer; $\mathrm{V}$ - retention volume of the marker ligand, when its specific concentration is completely suppressed, e.g. by a high concentration of the displacer; $\mathrm{K}_{\mathrm{dDis}}-$ dissociation constant of the displacer; $\mathrm{P}$ - product of the $\mathrm{B}_{\max }$ (the number of the available binding sites) and $\left(\mathrm{K}_{\mathrm{dDis}} / \mathrm{K}_{\mathrm{dmarker}}\right), \mathrm{K}_{\mathrm{dmarker}}-$ dissociation constant of the marker ligand [11]. 

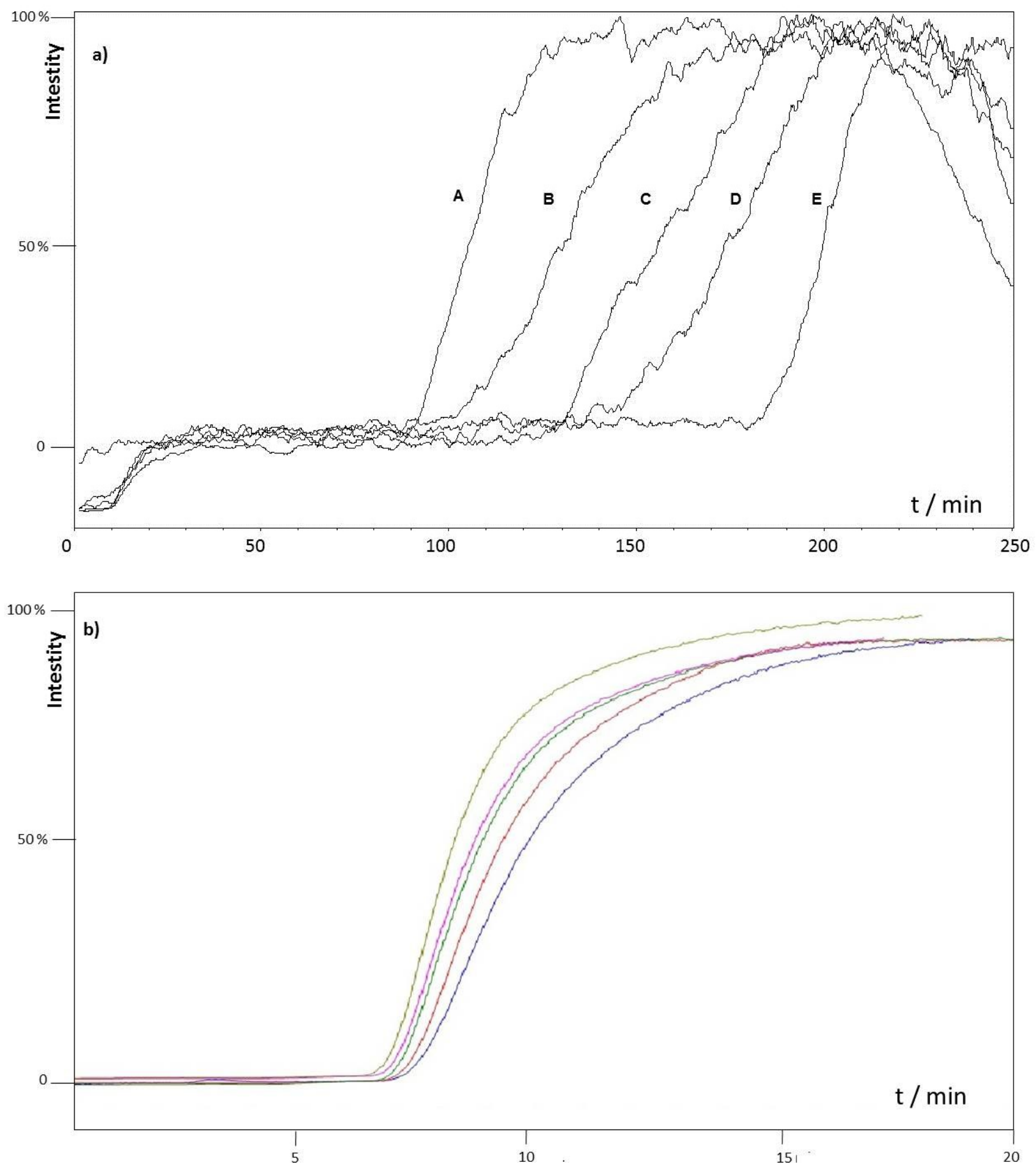

Figure 5. Frontal chromatograms of (a) increasing concentrations of $\left[{ }^{3} \mathrm{H}\right]$-epibatidine $(A=240 \mathrm{pM}, \mathrm{B}=150 \mathrm{pM}$, $C=100 \mathrm{pM}, \mathrm{D}=80 \mathrm{pM}$ and $\mathrm{E}=60 \mathrm{pM}$ ) on the $\alpha_{3} \beta_{4} \alpha_{5}$ nicotinic receptor column and (b) increasing concentrations of mecamylamine on $\alpha_{3} \beta_{4}$ nicotinic receptor column. For details please refer to the paper by Ciesla et al. [30]. With permission from [30]

This approach was for example used to determine $K_{d}$ values of several alkaloids binding to $\alpha_{3} \beta_{4}$ and $\alpha_{3} \beta_{4} \alpha_{5}$ nicotinic receptors [30].

Another chromatographic mode that can be used to study interactions between the adsorbed transmembrane receptors and ligands is nonlinear chromatography [11]. In this approach, the investigated small molecular compounds are injected onto a CMAC column close to, or at target saturation. The observed peak asymmetry allows the study of the kinetics and thermodynamics of interaction between the ligands and immobilized protein target. CMAC columns can be used as a first step to identify 
pharmacologically active secondary metabolites from natural mixtures and after isolation of these compounds, they can be applied to obtain pharmacokinetic and pharmacodynamic data.

In zonal chromatography, a small amount of the ligand is loaded onto the column and this allows for ranking the affinity of the investigated compounds [11].

\section{The use of CMACs in identification of pharmacologically active metabolites from natural mixtures}

In the early reports of CMAC column usage, the columns were applied to the study of the binding characteristics of the immobilized transmembrane proteins [7]. However, CMAC columns can be successfully applied in the process of identification of pharmacologically active compounds present in complex natural matrices targeting transmembrane receptors $[7,46]$. There are several issues that need to be taken into consideration when applying CMACs in drug discovery from natural samples. The first decision that has to be made is in choosing extracts that will be screened using the newly developed bioassay. The samples to be run on the CMAC columns should not be random, but carefully selected. Plant extracts that previously were reported to exert desired pharmacological activity may be considered for the screening experiments. However, the observed biological effect may not be from the result of the interaction of secondary metabolites present in the extract and the targeted protein, immobilized on the artificial membrane support. Another idea of narrowing down the possible screening extract candidates is to use a simple in vivo model (C. elegans or D. melanogaster) and test the extracts for biological activity. The extracts that exert the biological effect are further screened to identify phytochemicals responsible for this effect. Again, there has to be a known, or at least a rightfully hypothesized connection between activation/inhibition of the targeted transmembrane protein and pharmacological activity tested in the in vivo model. The easiest way to check for the possible presence of phytochemicals interacting with the transmembrane receptors is to use frontal affinity chromatography and a marker ligand known to bind to the transmembrane receptor $[7,30]$. The displacement observed after co-injecting the analysed extract and the marker ligand would indicate the presence of phytochemicals competing with the marker for the binding site. This approach can be applied to screen extracts for compounds interacting with both orthosteric and allosteric binding sites. Figure 6 presents the displacement of mecamylamine, a known $\mathrm{nAChR}$ allosteric inhibitor displaced by Lycopodium clavatum L. extract on CMAC columns with immobilized two types of nicotinic receptors $\alpha_{3} \beta_{4}$ and $\alpha_{3} \beta_{4} \alpha_{5}$ [30].

The identification process of individual secondary metabolites responsible for the observed displacement of the marker ligand is performed using the "missing peak chromatography" approach [46]. One of the greatest challenges in drug discovery from plant extracts, using CMAC columns, is discerning specific interactions (phytochemicals interacting with the immobilized transmembrane target) from nonspecific interactions (compounds interacting with IAM particles or other transmembrane proteins coimmobilized with the studied targeted receptor). Lipophilic compounds have been found to be strongly retained on IAM.PC particles and an alternative approach has been proposed to study such ligands: immobilization of cell membrane fragments onto the surface of an open tubular capillary (OT columns) [7]. For example, OT columns were successfully used to immobilize cell membrane fragments with CB1/CB2 receptors and further for the identification of endocannabinoid receptor ligands from the extract of Zanthoxylum clava-herculis L. [34, 47]. The development and the use of OT columns remain beyond the scope of this review and interested readers are encouraged to refer to other publications [7]. Coimmobilization of other proteins does not need to be considered as a CMAC disadvantage, as the same column can be utilized to study compounds binding to multiple transmembrane proteins [28]. To 
characterize multiple proteins co-immobilized on the IAM particles, different marker ligands have to be used, that specifically bind to the receptors of interest. Every receptor has to be characterized in separate runs.
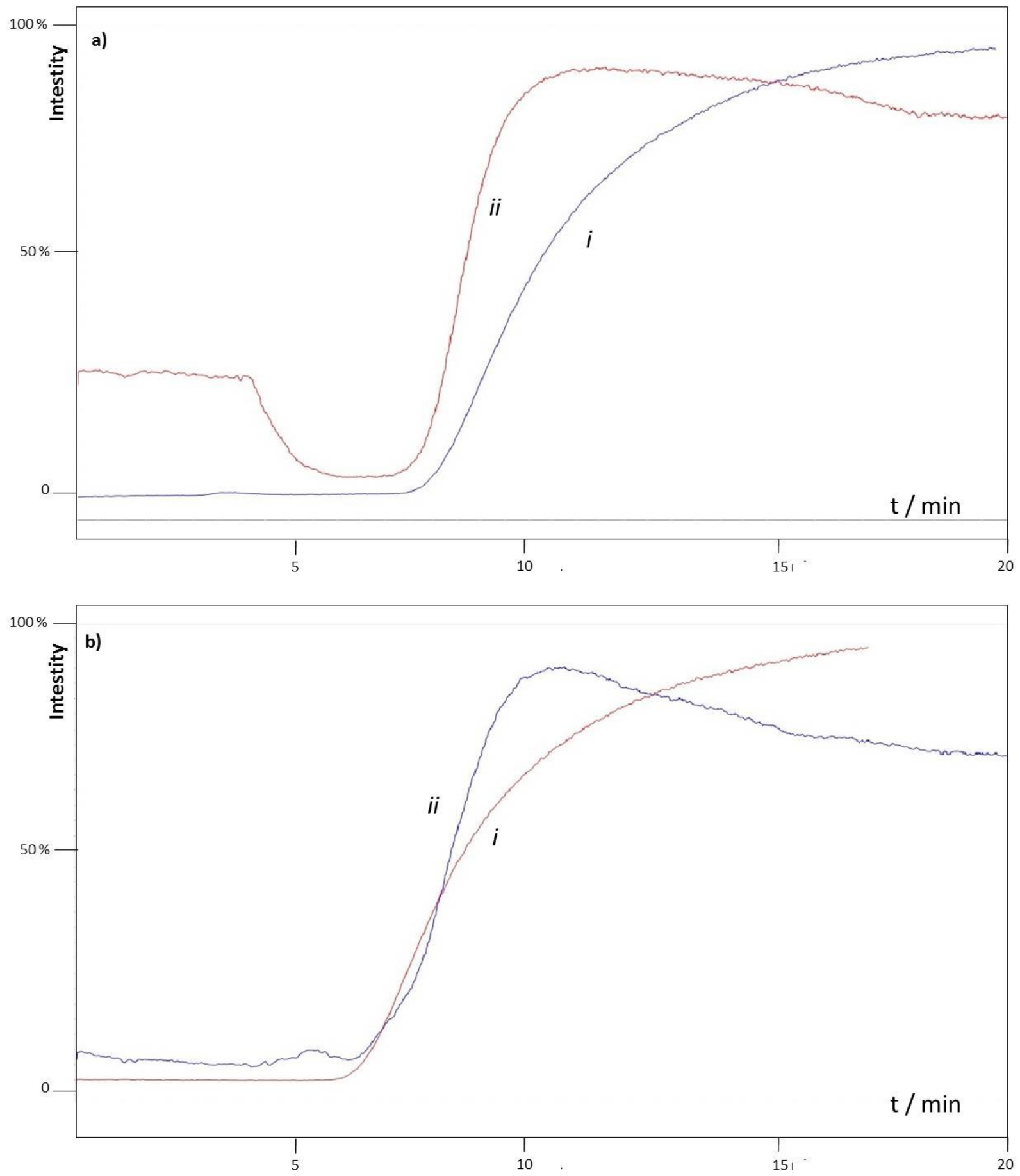

Figure 6. Frontal elution profile of $1 \mu \mathrm{M}$ mecamylamine (I) and $1 \mu \mathrm{M}$ mecamylamine $+5 \%$ Lycopodium clavatum (ii) on (a) the $\alpha_{3} \beta_{4}$ nicotinic receptor column and (b) $\alpha_{3} \beta_{4} \alpha_{5}$ nicotinic receptor column. For details please refer to the paper by Ciesla et al. [30]. With permission from [30].

The missing peak chromatography approach requires the use of a negative control CMAC column, CMAC(-). As previously stated, transfected cell lines stably overexpressing studied transmembrane proteins are recommended for the preparation of CMAC columns. A negative control column (CMAC(-)) is prepared using the same cell line, however not expressing the receptors overexpressed by the transfected line. For example, in the case of nAChR CMAC columns, HEK cells overexpressing desired nAChR subtypes were used 
to prepare $\mathrm{CMAC}(+)$, while HEK 293 cells were utilized to develop $\mathrm{CMAC}(-)$ [30]. In the missing peak chromatography technique, plant extracts are chromatographed on $\mathrm{CMAC}(+)$ and $\mathrm{CMAC}(-)$ columns using ammonium acetate buffer $(10 \mathrm{mM}, \mathrm{pH} 7.4)$ delivered at $0.2 \mathrm{ml} / \mathrm{min}$ as mobile phase [46]. Fractions are collected based on the observed chromatograms. The obtained fractions are subsequently fingerprinted on C18 columns and the fingerprints obtained using $\mathrm{CMAC}(+)$ and $\mathrm{CMAC}(-)$ are compared. The difference between the $\mathrm{CMAC}(+)$ and $\mathrm{CMAC}(-)$ columns is in the availability of the immobilized protein target. The differences in the retention of a compound on the $C M A C(+)$ column relative to the CMAC(-) column is due to specific interactions with the target $[7,46]$. A peak that is missing in the fingerprint of a $\mathrm{CMAC}(+)$ early fraction, but present in the control and corresponding CMAC(-) early fraction, is considered to bind to the immobilized transmembrane protein target on the $\mathrm{CMAC}(+)$ column [46]. Elution of this particular compound in a later fraction of $\mathrm{CMAC}(+)$, along with its absence in the corresponding fraction in $\mathrm{CMAC}(-)$, is a further confirmation of the affinity of this compound for the targeted transmembrane receptor in CMAC(+) [46]. Compounds identified as specifically binding to the studied receptors can be subsequently isolated using analytical or semipreparative HPLC, their structures elucidated and further tested in functional assays or in vivo models.

CMAC chromatography columns with immobilized cell membrane fragments on IAM particles do not solve all of the problems in the drug discovery process from natural samples. This approach requires the use of negative control columns and the solvent choice is limited to buffered aqueous solvents (with only up to approx. $10 \%$ of organic modifier). CMAC columns, however, do speed up the process of target identification, without the need to isolate individual compounds and test them separately using different assays. CMAC is an assay, in which transmembrane proteins remain submerged in a natural lipid environment, in close to physiological conditions. CMAC columns allow for the identification of compounds binding to receptor subtypes. After identification and isolation of pharmacologically active phytochemicals, CMAC columns may be used to study their interaction with the immobilized protein target (obtaining pharmacodynamics and pharmacokinetic data). The drug discovery process with CMACs is a targetoriented technique, ideally suited for creating libraries of natural compounds interacting with a specific cell membrane target.

\section{Future directions}

The potential of cell membrane affinity chromatography columns in the drug discovery process from natural samples has been underestimated. Pharmaceutical companies have focused their discovery programs on automated high-throughput screening techniques (HTS), which are not suitable for complex matrices. However, the introduction of HTS techniques did not result in the predicted substantial increase of new classes of drugs entering the market [8]. Secondary metabolites are evolutionary-designed compounds targeting specific, often highly-conserved signalling pathways and are already optimized to be pharmacologically active. One of the ways of unravelling the mysteries of natural compounds is to develop approaches enabling identification of these phytochemicals. Cellular membrane affinity chromatography columns allow phytochemists to selectively identify compounds interacting with transmembrane proteins. We predict CMAC columns have a potential to become the next generation of drug discovery tools in phytochemistry and pharmacology labs. These columns may be easily applied to build libraries of natural compounds that can move drug discovery projects forward to the next step. Some advice is given: carefully choose your transmembrane target by closely studying the possible pathways involved in the disease. An example is given from our lab: we are interested in cellular signalling pathways that have evolved to protect neurons from different forms of biological stress (oxidative, metabolic, proteotoxic or 
inflammatory stress). Published data indicated that the activation of the BDNF (brain-derived neurotrophic factor) pathway is neuroprotective [48]. BDNF works by binding with transmembrane tropomyosin receptor kinase (TrkB). A CMAC column with the immobilized TrkB receptors is to be used to screen selected plant extracts for natural compounds binding to this receptor and mimicking the neuroprotective effects of BDNF. Preparation of CMAC columns appears to be considered as a complicated approach and this may prevent it from common use in drug discovery or phytochemistry laboratories. The truth is, the preparation of CMAC columns is possible in almost every lab focusing on natural product discovery. Future direction may include the development of a kit that would allow interested labs to easily develop a column with the immobilized desired transmembrane protein.

CMAC has a potential to work well with other drug discovery approaches, for example, ligand fishing techniques. Pharmacologically active compounds may be first identified using ligand fishing techniques followed by ligand-protein interaction studies with a CMAC column.

The advent of novel drug discovery approaches is needed in this new era when drug discovery from natural samples is again becoming popular. The use of old techniques, that were one of the reasons for abandoning natural product screening, need to be replaced by innovative assays. Cellular membrane affinity chromatography together with ligand fishing techniques have everything that is required to become drug discovery techniques of the $21^{\text {st }}$ century.

\section{References}

[1] M.P. Mattson. What Doesn't Kill You. Sci Am 313 (2015) 40-45.

[2] M.P. Mattson, A. Cheng. Neurohormetic phytochemicals: Low-dose toxins that induce adaptive neuronal stress responses. Trends Neurosci 29 (2006) 632-639.

[3] M.P. Mattson, T.G. Son, S. Camandola. Viewpoint: mechanisms of action and therapeutic potential of neurohormetic phytochemicals. Dose Response 5 (2007) 174-186.

[4] V. Murugaiyah, M.P. Mattson. Neurohormetic phytochemicals: An evolutionary-bioenergetic perspective. Neurochem Int 89 (2015) 271-280.

[5] J. Lee, D.G. Jo, D. Park, H.Y. Chung, M.P. Mattson. Adaptive cellular stress pathways as therapeutic targets of dietary phytochemicals: focus on the nervous system. Pharmacol Rev 66 (2014) 815-868.

[6] B. Shen. A New Golden Age of Natural Products Drug Discovery. Cell 163 (2015) 1297-1300.

[7] L. Ciesla, R. Moaddel. Comparison of analytical techniques for the identification of bioactive compounds from natural products. Nat Prod Rep 33 (2016) 1131-1145.

[8] A.L. Harvey, R. Edrada-Ebel, R.J. Quinn. The re-emergence of natural products for drug discovery in the genomics era. Nat Rev Drug Discov 14 (2015) 111-129.

[9] M. Yasuda, D.R. Wilson, S.D. Fugmann, R. Moaddel. Synthesis and characterization of SIRT6 protein coated magnetic beads: identification of a novel inhibitor of SIRT6 deacetylase from medicinal plant extracts. Anal Chem 83 (2011) 7400-7407.

[10] N. Singh, S. Ravichandran, D.D. Norton, S.D. Fugmann, R. Moaddel. Synthesis and characterization of a SIRT6 open tubular column: predicting deacetylation activity using frontal chromatography. Anal Biochem 436 (2013) 78-83.

[11] R. Moaddel, I.W. Wainer. The preparation and development of cellular membrane affinity chromatography columns. Nat Protoc 4 (2009) 197-205.

[12] E. Brekkan, A. Lundqvist, P. Lundahl. Immobilized membrane vesicle or proteoliposome affinity chromatography. Frontal analysis of interactions of cytochalasin B and D-glucose with the human red cell glucose transporter. Biochemistry 35 (1996) 12141-12145. 
[13] Y. Zhang, Y. Xiao, K.J. Kellar, I.W. Wainer. Immobilized nicotinic receptor stationary phase for on-line liquid chromatographic determination of drug-receptor affinities. Anal Biochem 264 (1998) 22-25.

[14] S. Ong, H. Liu, X. Qiu, G. Bhat, C. Pidgeon. Membrane partition coefficients chromatographically measured using immobilized artificial membrane surfaces. Anal Chem 67 (1995) 755-762.

[15] I.W. Wainer, Y. Zhang, Y. Xiao, K.J. Kellar. Liquid chromatographic studies with immobilized neuronal nicotinic acetylcholine receptor stationary phases: effects of receptor subtypes, $\mathrm{pH}$ and ionic strength on drug-receptor interactions. J Chromatogr B Biomed Sci Appl 724 (1999) 65-72.

[16] M.T. Baynham, S. Patel, R. Moaddel, I.W. Wainer. Multidimensional on-line screening for ligands to the alpha3beta4 neuronal nicotinic acetylcholine receptor using an immobilized nicotinic receptor liquid chromatographic stationary phase. J Chromatogr B Analyt Technol Biomed Life Sci 772 (2002) 155-161.

[17] K. Jozwiak, J. Haginaka, R. Moaddel, I.W. Wainer. Displacement and nonlinear chromatographic techniques in the investigation of interaction of noncompetitive inhibitors with an immobilized alpha3beta4 nicotinic acetylcholine receptor liquid chromatographic stationary phase. Anal Chem $\mathbf{7 4}$ (2002) 4618-4624.

[18] R. Moaddel, I.W. Wainer. Immobilized nicotinic receptor stationary phases: going with the flow in high-throughput screening and pharmacological studies. J Pharm Biomed Anal 30 (2003) 1715-1724.

[19] K. Jozwiak, S.C. Hernandez, K.J. Kellar, I.W. Wainer. Enantioselective interactions of dextromethorphan and levomethorphan with the alpha 3 beta 4-nicotinic acetylcholine receptor: comparison of chromatographic and functional data. J Chromatogr B Analyt Technol Biomed Life Sci 797 (2003) 373-379.

[20] K. Jozwiak, S. Ravichandran, J.R. Collins, I.W. Wainer. Interaction of noncompetitive inhibitors with an immobilized alpha3beta4 nicotinic acetylcholine receptor investigated by affinity chromatography, quantitative-structure activity relationship analysis, and molecular docking. J Med Chem 47 (2004) 4008-4021.

[21] R. Moaddel, K. Jozwiak, R. Yamaguchi, C. Cobello, K. Whittington, T.K. Sarkar, S. Basak, I.W. Wainer. On-line screening of conformationally constrained nicotines and anabasines for agonist activity at the alpha3beta4- and alpha4beta2-nicotinic acetylcholine receptors using immobilized receptorbased liquid chromatographic stationary phases. J Chromatogr B Analyt Technol Biomed Life Sci $\mathbf{8 1 3}$ (2004) 235-240.

[22] R. Moaddel, K. Jozwiak, K. Whittington, I.W. Wainer. Conformational mobility of immobilized alpha3beta2, alpha3beta4, alpha4beta2, and alpha4beta4 nicotinic acetylcholine receptors. Anal Chem 77 (2005) 895-901.

[23] R. Moaddel, K. Jozwiak, R. Yamaguchi, I.W. Wainer. Direct chromatographic determination of dissociation rate constants of ligand-receptor complexes: assessment of the interaction of noncompetitive inhibitors with an immobilized nicotinic acetylcholine receptor-based liquid chromatography stationary phase. Anal Chem 77 (2005) 5421-5426.

[24] K. Jozwiak, R. Moaddel, R. Yamaguchi, A. Maciuk, I.W. Wainer. Non-competitive inhibitory activities of morphinan and morphine derivatives at the alpha 3 beta 4 Neuronal nicotinic acetylcholine receptor determined using nonlinear chromatography and chemometric techniques. Pharm Res $\mathbf{2 3}$ (2006) 2175-2182.

[25] R. Moaddel, K. Jozwiak, I.W. Wainer. Allosteric modifiers of neuronal nicotinic acetylcholine receptors: new methods, new opportunities. Med Res Rev 27 (2007) 723-753.

[26] K. Jozwiak, S. Ravichandran, J.R. Collins, R. Moaddel, I.W. Wainer. Interaction of noncompetitive inhibitors with the alpha3beta2 nicotinic acetylcholine receptor investigated by affinity chromatography and molecular docking. J Med Chem 50 (2007) 6279-6283.

[27] R. Moaddel, R.V. Oliveira, T. Kimura, P. Hyppolite, M. Juhaszova, Y. Xiao, K.J. Kellar, M. Bernier, I.W. Wainer. Initial synthesis and characterization of an alpha7 nicotinic receptor cellular membrane affinity chromatography column: effect of receptor subtype and cell type. Anal Chem 80 (2008) 4854. 
[28] T. Kitabatake, R. Moaddel, R. Cole, M. Gandhari, C. Frazier, J. Hartenstein, A. Rosenberg, M. Bernier, I.W. Wainer. Characterization of a multiple ligand-gated ion channel cellular membrane affinity chromatography column and identification of endogenously expressed receptors in astrocytoma cell lines. Anal Chem 80 (2008) 8673-8680.

[29] R. Moaddel, J.F. Cloix, G. Ertem, I.W. Wainer. Multiple receptor liquid chromatographic stationary phases: the co-immobilization of nicotinic receptors, gamma-amino-butyric acid receptors, and $\mathrm{N}$ methyl D-aspartate receptors. Pharm Res 19 (2002) 104-107.

[30] L. Ciesla, M. Okine, A. Rosenberg, K.S.S. Dossou, L. Toll, I.W. Wainer, R. Moaddel. Development and characterization of the alpha3beta4alpha5 nicotinic receptor cellular membrane affinity chromatography column and its application for on line screening of plant extracts. J Chromatogr $A$ 1431 (2016) 138-144.

[31] F. Beigi, I.W. Wainer. Syntheses of immobilized G protein-coupled receptor chromatographic stationary phases: characterization of immobilized mu and kappa opioid receptors. Anal Chem 75 (2003) 4480-4485.

[32] F. Beigi, K. Chakir, R.P. Xiao, I.W. Wainer. G-protein-coupled receptor chromatographic stationary phases. 2. Ligand-induced conformational mobility in an immobilized beta2-adrenergic receptor. Anal Chem 76 (2004) 7187-7193.

[33] R. Moaddel, H.K. Musyimi, M. Sanghvi, C. Bashore, C.R. Frazier, M. Khadeer, P. Bhatia, I.W. Wainer. Synthesis and characterization of a cellular membrane affinity chromatography column containing histamine 1 and P2Y(1) receptors: a multiple G-protein coupled receptor column. J Pharm Biomed Anal 52 (2010) 416-419.

[34] R. Moaddel, A. Rosenberg, K. Spelman, J. Frazier, C. Frazier, S. Nocerino, A. Brizzi, C. Mugnaini, I.W. Wainer. Development and characterization of immobilized cannabinoid receptor (CB1/CB2) open tubular column for on-line screening. Anal Biochem 412 (2011) 85-91.

[35] I. Gonzalez-Mariscal, S.M. Krzysik-Walker, M.E. Doyle, Q.R. Liu, R. Cimbro, S. Santa-Cruz Calvo, S. Ghosh, L. Ciesla, R. Moaddel, O.D. Carlson, R.P. Witek, J.F. O'Connell, J.M. Egan. Human CB1 Receptor Isoforms, present in Hepatocytes and beta-cells, are Involved in Regulating Metabolism. Sci Rep 6 (2016) 33302.

[36] R. Moaddel, L. Lu, M. Baynham, I.W. Wainer. Immobilized receptor- and transporter-based liquid chromatographic phases for on-line pharmacological and biochemical studies: a mini-review. J Chromatogr B Analyt Technol Biomed Life Sci 768 (2002) 41-53.

[37] R. Moaddel, P.L. Bullock, I.W. Wainer. Development and characterization of an open tubular column containing immobilized P-glycoprotein for rapid on-line screening for P-glycoprotein substrates. J Chromatogr B Analyt Technol Biomed Life Sci 799 (2004) 255-263.

[38] R. Moaddel, R. Hamid, S. Patel, P.L. Bullock, I.W. Wainer. Identification of P-glycoprotein substrates using open tubular chromatography on an immobilized P-glycoprotein column: Comparison of chromatographic results with Caco-2 permeability. Anal Chim Acta 578 (2006) 25-30.

[39] K.L. Habicht, N.S. Singh, M.A. Khadeer, R. Shimmo, I.W. Wainer, R. Moaddel. Characterization of a multiple endogenously expressed adenosine triphosphate-binding cassette transporters using nuclear and cellular membrane affinity chromatography columns. J Chromatogr A 1339 (2014) 80-85.

[40] S. Wang, M. Sun, Y. Zhang, H. Du, L. He. A new A431/cell membrane chromatography and online high performance liquid chromatography/mass spectrometry method for screening epidermal growth factor receptor antagonists from Radix sophorae flavescentis. J Chromatogr A 1217 (2010) 52465252.

[41] L.C. He, S.C. Wang, G.D. Yang, Y.M. Zhang, C.H. Wang, B.X. Yuan, X.F. Hou. Progress in cell membrane chromatography. Drug Discov Ther 1 (2007) 104-107.

[42] S. Han, Y. Lv, F. Wei, J. Fu, Q. Hu, S. Wang. Screening of bioactive components from traditional Chinese medicines using cell membrane chromatography coupled with mass spectrometry. Phytochem Anal (2018). 
[43] Y. Lin, C. Wang, Y. Hou, W. Sun, D. Che, L. Yang, T. Zhang, M. Sun, H. HeHe, L. He. Simultaneous identification of three pseudo-allergic components in Danshen injection by using high-expression Mas-related $\mathrm{G}$ protein coupled receptor X2 cell membrane chromatography coupled online to HPLCESI-MS/MS. J Sep Sci (2018).

[44] K.L. Habicht, N.S. Singh, F.E. Indig, I.W. Wainer, R. Moaddel, R. Shimmo. The development of mitochondrial membrane affinity chromatography columns for the study of mitochondrial transmembrane proteins. Anal Biochem 484 (2015) 154-161.

[45] E.S. Ng, N.W. Chan, D.F. Lewis, O. Hindsgaul, D.C. Schriemer. Frontal affinity chromatography-mass spectrometry. Nat Protoc 2 (2007) 1907-1917.

[46] A. Maciuk, R. Moaddel, J. Haginaka, I.W. Wainer. Screening of tobacco smoke condensate for nicotinic acetylcholine receptor ligands using cellular membrane affinity chromatography columns and missing peak chromatography. J Pharm Biomed Anal 48 (2008) 238-246.

[47] K.S. Dossou, K.P. Devkota, C. Morton, J.M. Egan, G. Lu, J.A. Beutler, R. Moaddel. Identification of CB1/CB2 ligands from Zanthoxylum bungeanum. J Nat Prod 76 (2013) 2060-2064.

[48] M.P. Mattson, K. Moehl, N. Ghena, M. Schmaedick, A. Cheng. Intermittent metabolic switching, neuroplasticity and brain health. Nat Rev Neurosci 19 (2018) 63-80. 